\title{
Respiratory viral infections as promoters of allergic sensitization and asthma in animal models
}

\author{
J. Schwarze*, E.W. Gelfand ${ }^{\#}$
}

Respiratory viral infections as promoters of allergic sensitization and asthma in animal models. J. Schwarze, E.W. Gelfand. (C) ERS Journals Ltd 2002.

ABSTRACT: Respiratory virus infections can trigger exacerbations of asthma and may also contribute to allergic sensitization to aeroallergens and the development of asthma. Conversely, atopy may predispose to more severe virus-induced airway disease. The animal models reviewed in this article support the hypothesis that respiratory virus infections can promote allergic sensitization and the development of asthma. Respiratory viruses can prevent induction of tolerance and enhance sensitization to inhaled allergens resulting in increased airway inflammation and airway hyperresponsiveness.

Probable mechanisms involved in this enhanced sensitization are increased permeability of the airway mucosa to allergens and recruitment of dendritic cells to the respiratory epithelium during acute infection. Factors involved in augmenting the consequences of allergic airway sensitization appear to be T-cells, especially CD8+ T-cells as regulators of this process, interleukin-5 as a pivotal cytokine for eosinophilic airway inflammation and eosinophils themselves as effector cells triggering airway hyperresponsiveness. Depending on the timing of allergen exposure, respiratory virus infections which elicit a significant type 1 T-helper cell cytokine response may also downregulate allergic sensitization.

Respiratory virus infections in animals previously sensitized to aeroallergens result in prolonged increases in inflammation and airway responsiveness, indicating that critical interactions between immune responses to allergen sensitization and the responses to infection can lead to more severe disease.

Taken together, animal models have proved valuable in generating a number of plausible pathogenetic concepts, and can be used to address a host of unresolved questions regarding the immunology of respiratory virus infections, allergic sensitization and asthma.

Eur Respir J 2002; 19: 341-349.

*Children's Hospital, St Josef-Hospital Bochum, Ruhr-University Bochum, Bochum, Germany and ${ }^{\sharp}$ Division of Cell Biology, Department of Pediatrics, National Jewish Medical and Research Center, Denver, CO, USA.

Correspondence: J. Schwarze, Klinik für Kinder- und Jugendmedizin im St Josef-Hospital Bochum, Alexandrinenstr. 5, 44791 Bochum, Germany. Fax: 492345092612

Email: juergen.schwarze@

ruhr-uni-bochum.de

\section{Keywords: Airway inflammation,} airway obstruction, allergic sensitization, animal models, asthma, respiratory virus infection

Received: January 152001

Accepted after revision June 202001

This study was supported by Grants HL-61005 and HL-36577 (E.W. Gelfand) from the National Institutes of Health, Grant Schw 597/1-1 from the Deutsche Forschungsgemeinschaft and Grant 01GC9802 (J. Schwarze) from the Bundesministerium für Bildung und Forschung.

\section{Epidemiological background}

Asthma in childhood is associated in most cases with sensitization to inhaled allergens and allergic airway inflammation. Onset of asthma has been associated with viral lower respiratory tract infections [1]. Further, acute viral respiratory infections can trigger wheezing and exacerbations of asthma in children and adults [2,3]. Indeed, $80 \%$ of wheezing episodes in schoolchildren have been associated with viral respiratory tract infections, most frequently rhinovirus infection [4].

In infants and toddlers under the age of $2 \mathrm{yrs}$, the virus most frequently isolated during wheezing episodes is respiratory syncytial virus (RSV) $[5,6]$. There is epidemiological evidence suggesting that respiratory viruses, especially RSV, may not only trigger asthma exacerbations but also contribute to or facilitate allergic sensitization to aeroallergens and subsequently the development of asthma. FRICK et al. [7] observed coincidence of respiratory infections and the onset of allergic sensitization to aeroallergens in infants with a family history of allergy. In these children, allergic sensitization could be first detected 1-2 months following a viral respiratory tract infection. A close link, particularly with RSV-induced bronchiolitis and development of asthma, has been identified in several studies [8-11]. In a recent prospective cohort study with matched controls, SIGURS et al. [12] identified RSV bronchiolitis in

Previous articles in this series: No. 1: von Mutius E. Infection: friend or foe in the development of atopy and asthma? The epidemiological evidence. Eur Respir J 2001; 18: 872-881. No. 2: Wennergren G, Kristjánsson S. Relationship between respiratory syncytial virus bronchiolitis and future obstructive airway diseases. Eur Respir $J$ 2001; 18: 1044-1058. No. 3: Renz H, Herz U. The bidirectional capacity of bacterial antigens to modulate allergy and asthma. Eur Respir J 2002; 19: 158-171. 
infancy as the most important risk factor for the subsequent development of asthma and sensitization to common allergens by the age of $3 \mathrm{yrs}$. This risk increased further if there was a family history of asthma or atopy. A follow-up report showed that by the age of 7 yrs allergic sensitization and asthma were still more prevalent in the group of children who had had RSV bronchiolitis in the first year of life than in controls [13]. Other studies have not demonstrated an association between RSV bronchiolitis and allergic sensitization $[8,11,14]$.

A recent quantitative meta-analysis of studies investigating associations of RSV disease in infants with the development of asthma in childhood confirmed an increased risk of recurrent wheezing during the first 5 yrs of life, but not thereafter [15]. An association of RSV infection with a personal or family history of atopy was not detected. Due to the heterogeneity of the studies analysed, the relationship between RSV infection and allergic sensitization, as assessed by skin-prick test, could not be evaluated. In addition, this meta-analysis did not include the 7-yr follow-up of Sigurs et al.[13] and did not take into account the fact that only this group used a prospectively controlled longitudinal cohort. All of the other studies used retrospective control groups.

One criticism of the epidemiological studies discussed earlier has been their selection of children with RSV disease severe enough to require hospitalization. Selection of this small minority of RSV-infected children constitutes a bias that probably selects for predisposition to severe RSV disease, such as in atopy. The study of STEIN et al. [16] that utilized the large birth cohort from the Tucson Children's Respiratory Study does not have this selection bias. Here, less severe RSV infection during the first 3 yrs of life was found to be a transient risk factor for wheezing at 6 yrs, but this did not persist at 13 yrs. RSV infection was not associated with an increased risk of allergic sensitization in this population, but such an association may have been obscured by the unusually high rate of allergic sensitization in this cohort. Cumulatively, these observations suggest that RSV infection can facilitate or enhance allergic sensitization and the development of allergic airway disease, at least in some patients.

\section{Animal studies}

In order to investigate some of the underlying immunological mechanisms involved in the interplay of lower respiratory tract infections with respiratory viruses, allergic airway sensitization, and the development of obstructive airway disease and asthma, several animal models have been utilized. In these models, a range of different respiratory viruses have been assessed, including RSV, parainfluenza virus (PIV) 3 and influenza, which differ in their impact on the development of obstructive airway disease in humans. These viruses are all useful in determining the mechanisms of interaction between lower respiratory tract virus infection and allergic sensitization of the airways, although observations obtained with one virus may not be transferable to other viruses. The animal models reviewed in the present article address the following issues and aim to reveal the pathogenetic mechanisms involved: 1) enhancement of allergic sensitization by respiratory virus infection; 2) increased airway inflammation and airway responsiveness as consequences of allergic airway sensitization following respiratory virus infection; and 3) increased respiratory virus-induced airway inflammation and responsiveness in established allergic airway sensitization.

\section{Enhancement of allergic sensitization by respiratory viral infections}

Since the 1980s, researchers have studied animal models to test the hypothesis that infection with respiratory viruses can trigger and enhance allergic sensitization to inhaled antigens, as had been reported for children [7]. Initially, the focus was on allergenspecific antibodies. SAKAMOTO et al. [17] infected mice with influenza virus at doses high enough to result in bronchopneumonia. Following infection, the mice were sensitized to an aerosol of ovalbumin (OVA) complexed with aluminium hydroxide adjuvant, and OVA-specific immunoglobulin (Ig)-E levels were titrated by passive cutaneous anaphylaxis in rats. Influenza infection prior to sensitization resulted in elevated OVA-specific IgE levels provided that the OVA aerosol exposure occurred during the acute infection on days 2-6 after infection. Sensitization 14 days after infection was not enhanced in this model. More recent studies have confirmed these observations using parallel models extended by an additional allergen challenge 3-4 weeks after primary sensitization [18, 19]. Yамамото et al. [19] reported a transient increase in airway dendritic cell number from day 2 to day 5 of influenza infection. In mice sensitized to OVA aerosol at the time of infection, the increase in dendritic cell number persisted for up to 5 weeks and was associated with high major histocompatibility complex class II expression by these cells.

The authors concluded that recruitment of dendritic cells, as antigen-presenting cells, to the airways during influenza infection may have contributed to enhanced sensitization to aeroallergens.

\section{Influenza infection prevents induction of tolerance to allergens}

Holt et al. [20] studied the effects of influenza infection in a model of tolerance induction. If mice were exposed to OVA aerosol prior to intraperitoneal sensitization with OVA and aluminium hydroxide, reduced levels of OVA-specific IgE and increased levels of OVA-specific IgG were detected. This phenomenon was interpreted as tolerance. Influenza infection at the time of OVA aerosol exposure prevented the development of tolerance, resulting in increased levels of OVA-specific IgE following 
subsequent intraperitoneal sensitization. Parallel findings were recently reported by TsiToura et al. [21]. They assessed the effects of influenza infection in a model of tolerance induction by intranasal application of OVA prior to intraperitoneal sensitization, which in turn was followed by intranasal OVA challenge. Influenza infection at the same time as the initial OVA application prevented tolerance induction, resulting in increased OVA-specific T-cell proliferation, increased OVA-specific IgE levels and increased type 2 T-helper cell (Th) cytokine production. When influenza infection did not coincide with the initial allergen exposure but preceded it by 15 or 30 days, tolerance was still prevented, as demonstrated by the lack of inhibition of OVA-induced T-cell proliferation. However, under these conditions, sensitization was associated with a strong Th1 immune response, with reduced production of interleukins (ILs)-4, -5 and -13 and increased production of interferon gamma (IFN- $\gamma$ ) upon restimulation with antigen. This Th1 response suppressed the production of OVA-specific IgE but resulted in enhanced OVA-specific IgG2a serum levels.

These findings suggest that infection with influenza prevents the development of tolerance and enhances IgE-mediated allergic sensitization if infection and sensitization coincide. If infection precedes primary allergen exposure, nonallergic Th1-driven sensitization is promoted.

Respiratory syncytial virus infection enhances sensitization to allergen

LeIbovitz et al. [22] and Freihorst et al. [23] studied the virus primarily implicated in the promotion of allergic sensitization via the airways and asthma development in children. They determined, in a murine model, the impact of infection with RSV on sensitization to inhaled allergens (ragweed and OVA) during acute infection. In both sets of experiments, RSV infection resulted in increased levels of allergen-specific antibodies in serum ( $\operatorname{IgE}$ and $\mathrm{IgG})$ and bronchoalveolar lavage (BAL) fluid (IgA and IgG). In a recent publication of $\mathrm{O}^{\prime}$ DonNELl et al. [24], induction of anaphylactic antibodies by respiratory viral infections was reported. Following infection with influenza or RSV and concomitant sensitization to OVA aerosol, but not after sensitization without infection, a large proportion of mice collapsed upon subsequent cutaneous challenge with the antigen. In mice susceptible to features of anaphylaxis, OVA-specific IgG1 was detected. This class of antibody, which is known to mediate anaphylaxis in mice [25], was not detected in mice which failed to react to cutaneous antigen challenge.

\section{Parainfluenza infection results in increased mucosal permeability}

In guinea-pig models of PIV 3 and RSV infection studied by RIEDEL et al. [26] and DAKhAMA et al. [27], this ability of respiratory viruses to increase production of allergen-specific antibodies was confirmed. In both sets of studies, sensitization was begun during acute infection, when increased permeability of the respiratory mucosa was demonstrated by RIEDEL et al.[26]. Serum levels of horseradish peroxidase were increased following inhalation of horseradish peroxidase aerosol by acutely infected mice compared to noninfected animals.

The investigators suggested that the increased permeability of the airway mucosa may be an important factor in virus-induced enhancement of sensitization via the airways.

These studies and additional data from a rat model of influenza infection [28] and bovine model of RSV infection [29] demonstrate that viral lower respiratory tract infections can enhance allergic sensitization to inhaled antigens and prevent the development of tolerance which may be induced by allergen exposure via the airways in the absence of infection (table 1).

Table 1. - Animal models of respiratory virus infection and allergic airway sensitization

\begin{tabular}{|c|c|c|c|c|}
\hline Animal & Respiratory virus & Allergen & Outcome & [Ref.] \\
\hline \multirow[t]{3}{*}{ Mouse } & Influenza & OVA & Enhanced sensitization & {$[17,18]$} \\
\hline & & & Failure of tolerance induction & {$[20,21]$} \\
\hline & & & Increased inflammation, AHR & {$[19,21]$} \\
\hline \multirow[t]{4}{*}{ Mouse } & Human RSV & OVA & Enhanced sensitization & {$[23]$} \\
\hline & & & Increased inflammation, AHR & {$[30]$} \\
\hline & & Ragweed & Enhanced sensitization & {$[22]$} \\
\hline & & $\begin{array}{l}\text { Dermatophagoides } \\
\text { farinae }\end{array}$ & Enhanced sensitization, prolonged AHR & [32] \\
\hline \multirow[t]{4}{*}{ Guinea-pig } & PIV 3 & OVA & Enhanced sensitization & [26] \\
\hline & & & Increased inflammation, AHR & {$[26]$} \\
\hline & & & No effect on inflammation or AHR & [33] \\
\hline & Human RSV & OVA & $\begin{array}{c}\text { Enhanced sensitization, increased } \\
\text { inflammation, AHR }\end{array}$ & [27] \\
\hline Rat & Influenza & OVA & $\begin{array}{c}\text { Enhanced sensitization, increased } \\
\text { inflammation, AHR }\end{array}$ & [28] \\
\hline Cow & Bovine RSV & $\begin{array}{l}\text { Micropolyspora } \\
\text { faeni }\end{array}$ & Enhanced sensitization & [29] \\
\hline
\end{tabular}

RSV: respiratory syncytial virus; PIV: parainfluenza virus; OVA: ovalbumin; AHR: airway hyperresponsiveness. 
Among the mechanisms involved, increased permeability of the airway mucosa to allergens and recruitment of dendritic cells to the airway epithelium during acute infection may be important, facilitating sensitization through increased antigen uptake and more effective antigen presentation. The effects of respiratory viruses on sensitization appear to be dependent on timing, due to changes in the quality of immune responses during the course of infection. As a result, infection with a given respiratory virus could potentially result in either promotion or inhibition of allergic sensitization.

\section{Enhanced consequences of allergic airway sensitization following respiratory virus infection}

\section{Guinea-pig models}

In addition to allergen-specific Igs as markers of sensitization, the consequences of virus-induced allergic airway sensitization have become a major focus of research based on the hypothesis that respiratory virus infections can predispose to the development of asthma. Airway inflammation and airway responsiveness to bronchoprovocation, both features of bronchial asthma, were first monitored in guinea-pig models of virus infection with allergen sensitization $[26,33]$. Following infection with both PIV 3 and RSV, enhanced sensitization to OVA aerosol was associated with increased airway inflammation, marked eosinophilia and airway hyperresponsiveness (AHR) to allergen challenge and to nonspecific provocation with methacholine [26, 27]. KUDLACZ and KNIPPENBERG [33], in contrast, did not find any consequences of enhanced sensitization following infection with PIV 3. In their model, PIV 3 infection prior to sensitization to OVA prevented the increases in histamine release, numbers of leukocytes in BAL fluid and methacholine-induced dyspnoea which were observed in noninfected sensitized guinea-pigs. The discrepancy between these observations and the findings reported by RIEDEL et al. [26] may be due to the fact that KudLACZ and KNIPPENBERG [33] used 10fold higher amounts of PIV 3 for infection, possibly inducing a long-lasting and significant Th1 response, which may have modified the initial sensitization response. Unfortunately, allergen-specific antibodies, as parameters of sensitization, were not monitored in this study.

\section{Murine models}

In mice, enhanced sensitization to aeroallergens following respiratory virus infection is also associated with increased airway inflammation and AHR. SuzuKi et al. [18] and Yамамото et al. [19] sensitized mice with OVA aerosol and aluminium hydroxide during the acute phase of influenza infection and then challenged them with the allergen 2-3 weeks after primary sensitization. In both studies, this resulted in increased OVA-specific IgE levels, increased airway inflammation and AHR compared to sensitization in the absence of infection or infection without sensitization. The composition of inflammatory cells was assessed in BAL fluid. Both groups observed a predominant increase in $\mathrm{CD} 8+\mathrm{T}$-cell numbers following sensitization in infected mice. In addition, YАмАмото et al. [19] also reported increases in numbers of CD4+ T-cells, eosinophils and macrophages.

The present authors have investigated a model of RSV infection and subsequent allergic sensitization to OVA via the airways in mice. In order to investigate whether RSV infection can predispose to allergen sensitization and the development of obstructive airway disease through modulation of immune responses, independently of mechanisms associated with the acute infection such as increased mucosal permeability, an approach differing from that of previously established models was chosen. In this approach, sensitization was begun only after the acute phase of the infection or even after resolution of the consequences of acute respiratory virus disease, i.e. on days $11-21$ or $21-30$ postinfection. Following RSV infection in mice, maximal viral replication occurs on day 4 and maximal inflammatory changes and AHR can be detected on days $6-8$. By day 21 , postinfection airway inflammation and AHR have completely resolved. Exposure to OVA aerosol alone on 10 consecutive days resulted in sensitization, as indicated by OVA-specific IgE and IgG1, but these mice did not develop altered airway responses to inhaled methacholine. Acute RSV infection prior to sensitization did not affect serum levels of allergen-specific antibodies [30]. This is in contrast to the models discussed earlier, in which exposure to allergen was begun during the acute phase of infection and resulted in increased allergen-specific antibody production.

Following RSV infection and subsequent exposure to allergen, airway responsiveness to nonspecific provocation with inhaled methacholine was assessed by barometric body plethysmography, monitoring "enhanced pause", a calculated value which correlates with airway resistance [34]. Twenty-four hours later, lungs and peribronchial lymph nodes (PBLNs), the regional lymph nodes of the lung, were harvested. Lung cells were isolated by collagenase digestion and inflammatory cells identified using light microscopy. Mononuclear cells from PBLNs were cultured and concentrations of the cytokines IFN- $\gamma$, IL-4 and IL-5 measured in supernatants by enzyme-linked immunosorbent assay (ELISA). RSV infection but not sham infection or administration of ultraviolet lightinactivated virus prior to sensitization resulted in lung eosinophilia and neutrophilia and the development of AHR to inhaled methacholine. These effects of RSV infection on subsequent exposure to allergen were associated with a decrease in IFN- $\gamma$ and increase in IL-4 production in cultures of mononuclear cells from PBLNs. IL-5 concentrations did not change significantly [30].

\section{Cytokines in respiratory syncytial virus-induced effects on allergic sensitization}

Due to the pivotal role of IL-5 in the development of airway eosinophilia and AHR, both in allergic 
sensitization [35-37] and acute RSV infection [38], the present authors sought to delineate the role of IL-5 in RSV-induced effects on allergic airway sensitization. In order to do this, both anti-IL-5 treatment and mice genetically deficient in IL-5 were studied. Treatment with anti-IL-5 during the allergen sensitization phase prevented eosinophil influx into the lungs and development of AHR [30]. This is in keeping with the effects of anti-IL-5 treatment reported in models of allergen-induced airway inflammation and AHR [35-37]. Anti-IL-5 treatment during the RSV infection phase alone significantly reduced lung eosinophilia and AHR following subsequent allergen exposure in the presence of IL-5 [39]. Mice genetically deficient in IL-5 failed to develop lung eosinophilia and AHR following RSV infection and allergen sensitization. Both were restored by administration of exogenous IL-5 during the acute infection. In contrast, reconstitution with IL-5 during the allergen sensitization phase alone did not restore AHR, despite inducing significant lung eosinophilia [39]. These data illustrate that the presence of IL-5, and possibly of eosinophils, during the acute infection phase is critical if not essential to the development of lung eosinophilia and AHR following subsequent allergen sensitization via the airways.

In order to further delineate the mechanisms involved, the roles of IL-4 and IFN- $\gamma$ were studied in the above model utilizing mice deficient in either of these cytokines. Mice deficient in IL-4 developed neither lung eosinophilia nor AHR following RSV infection and sensitization. The absence of IL-4 was compensated for by administration of IL-5, indicating that availability of IL-4 may be essential for IL-5 production, at least sufficient for the development of RSV-induced effects on airway sensitization [39]. IFN- $\gamma$ did not appear to contribute to the effects of RSV infection on allergen sensitization. Conversely, mice deficient in IFN- $\gamma$ developed increased lung eosinophilia and AHR, exceeding the effects of RSV infection and sensitization in wild-type controls [39].

\section{T-cells in respiratory syncytial virus-induced effects on allergic sensitization}

In order to define the role of T-cells as possible regulatory cells mediating the effects of RSV infection on airway allergen sensitization, two approaches were utilized: 1) depletion of T-cells during RSV infection and sensitization; and 2) adoptive transfer of T-cells which had been isolated from the PBLNs of RSVinfected mice and transferred (intravenously) to naive mice prior to allergen sensitization via the airways. Treatment of mice with anti-CD8 resulted in almost complete depletion of CD8+ T-cells (>96\%). This prevented the influx of neutrophils and eosinophils into the lungs and the development of AHR following allergen exposure. Depletion of CD4+ T-cells was also almost complete during RSV infection $(>98 \%)$, but could not be sustained during the allergen sensitization phase $(72 \%$ depletion). Although resulting in a reduction in lung eosinophilia and AHR, CD4+ T-cell depletion did not prevent these consequences of RSV infection on allergen sensitization. Adoptive transfer of CD3+ T-cells, harvested from PBLNs 14 days after infection resulted in eosinophil influx into the lungs and development of AHR associated with an increase in IL-5 production in noninfected recipients following allergen sensitization. In contrast, no such effects were observed following transfer of T-cells from noninfected mice or following transfer of $\mathrm{T}$-cells from RSV-infected animals into recipients without subsequent allergen sensitization. Further, the effects of RSV infection on allergen sensitization could also be transferred by CD8+ T-cells isolated from PBLNs. Transfer of isolated CD4+ T-cells, in contrast, did not result in the influx of eosinophils and AHR following allergen sensitization [40].

These observations indicate that T-cells, in particular CD8+ T-cells, are critical in mediating the effects of RSV infection on subsequent exposure of the airways to allergen. It may be speculated that IL-5-producing CD8+ T-cells (Tc2), play a pivotal role in this interaction, in contrast to the cytotoxic IFN- $\gamma$-producing CD8+ T-cells (Tc1), which dominate the acute response to viral infection between virus exposure and subsequent exposure to allergen. This former Tc2 subset may expand and become more dominant after the acute phase of the RSV infection has resolved, thus favouring eosinophilic airway inflammation and the development of AHR in response to allergic sensitization via the airways (fig. 1). This hypothesis needs to be tested by direct assessment of cytokine expression and production at the single cell level in T-cell subpopulations over the course of RSV infection and subsequent resolution.

Taken together, studies from a number of laboratories have demonstrated that enhanced sensitization to allergen following respiratory virus infections can be associated with increases in airway inflammation and airway responsiveness. In addition, RSV infection appears capable of enhancing the consequences of subsequent allergen sensitization independently of increasing levels of allergen-specific antibodies. The effects of RSV infection and also of infections with other respiratory viruses on allergic airway sensitization are probably mediated and regulated by T-cells

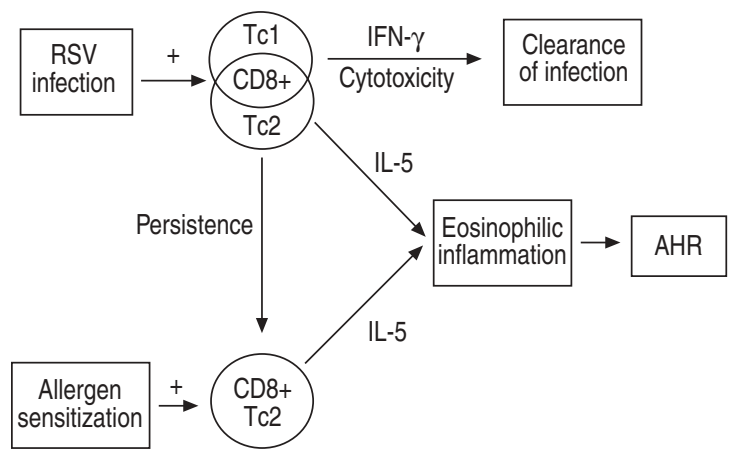

Fig. 1.-Role of CD8+ T-cells in virus-induced airway hyperresponsiveness (AHR) following acute infection and subsequent sensitization: a hypothesis. RSV: respiratory syncytial virus; Tc1: cytotoxic interferon gamma (IFN- $\gamma$ )-producing CD8+ T-cells; Tc2: noncytotoxic interleukin-5 (IL-5)-producing CD8+ T-cells; +: activation. 
and, under certain conditions, CD8+ T-cells. Interestingly, CD8+ T-cells are also pivotal in the development of AHR in both acute RSV infection [41] and allergic sensitization [42]. Further, the presence of IL-5 during acute RSV infection is essential for eosinophil influx into the airways and the development of AHR following subsequent allergen sensitization. In the light of these observations, it appears that RSV infection can prime and induce differentiation of a subset of CD8+ T-cells to produce IL-5 both during acute infection and following subsequent allergen exposure. This results in increased levels of IL-5 in the airways, favouring the development of eosinophilic inflammatory responses. The eosinophils, in turn, appear to be critical effector cells of the development of AHR, both in acute RSV infection and following subsequent allergic sensitization. A corollary of the studies in IL-4-deficient mice is that IL-4 in the lung milieu is essential for this capacity of CD8+ T-cells to differentiate into IL-5 producers.

\section{Respiratory virus-induced airway inflammation and responsiveness in established allergic airway sensitization}

The corollary of the issue of whether virus infections affect the response to allergen is whether allergen sensitization predisposes to more severe virusinduced disease. Individuals with airway allergies and asthma are prone to develop acute obstructive airway disease if infected with respiratory viruses. In addition, the question arises as to whether respiratory virus infections contribute to the perpetuation of established allergic airway disease and its severity. These issues have been addressed by only a few studies in animal models which have investigated the impact of respiratory virus infections on the development of airway inflammation and AHR in animals already sensitized to allergen.

\section{Increased airway inflammation and airway hyper-} responsiveness

RoBINSON et al. [43] studied the effects of RSV infection in guinea-pigs previously sensitized to OVA via the airways, monitoring airway responsiveness to acetylcholine and airway inflammation. Both sensitization alone and RSV infection alone resulted in AHR and airway inflammation with epithelial necrosis, airway wall oedema, mononuclear and granulocytic infiltrates, bronchoalveolar lymphoid tissue hyperplasia and goblet cell metaplasia. RSV infection in allergic animals further increased AHR and airway inflammation, particularly promoting airway epithelial necrosis. The authors concluded that prior sensitization potentiated the physiological and structural changes associated with RSV infection and posited that an established allergic diathesis may increase the severity of RSV infection in children.

\section{Prolonged airway hyperresponsiveness}

PeEbles et al. [31] utilized a mouse model of intraperitoneal sensitization to OVA with aluminium hydroxide followed by OVA aerosol challenge to assess the effects of RSV infection during the challenge. Monitoring airway responsiveness to methacholine at weekly intervals, they observed that RSV infection prolonged AHR. Eight days after infection, methacholine provocation resulted in AHR to the same degree in sensitized and challenged mice with or without RSV infection. However, on day 15 after infection, AHR was still present in mice that had been infected with RSV but not in those sensitized and challenged without infection. This prolongation of AHR was associated with an increase in the numbers of lymphocytes in the BAL fluid on day 15 . Eosinophils were the predominant cell type in the BAL fluid, but their numbers did not differ between RSV-infected and noninfected animals. In addition, on histological examination, increased alveolar and interstitial inflammation and increased mucus production were noted in mice infected with RSV. Prior allergen sensitization did not impact the virus load as determined by plaque assays from lung homogenates 4 days postinfection.

Recurrent respiratory syncytial virus infection in allergic airways

A study recently reported by MATSUSE et al. [32] focused on the effects of recurrent RSV infection in mice sensitized to Dermatophagoides farinae (Df). Following intraperitoneal sensitization (together with aluminium hydroxide), mice were challenged intranasally with the allergen 2, 6 and 14 weeks after sensitization. The day following each 3 -day challenge period, mice were infected with RSV or sham-infected. Controls were neither sensitized nor challenged and only infected with RSV or sham-infected. Following each challenge period, airway responsiveness to methacholine was measured using barometric body plethysmography. Primary RSV infection resulted in increased airway responsiveness in nonsensitized mice and lasted for 10 days. In these animals, the duration of AHR was shortened after the second RSV infection and was no longer detectable following tertiary infection. In contrast, RSV infection in mice sensitized to Df resulted in AHR that increased in duration following the secondary infection and was even more pronounced after tertiary infection. The magnitude of airway responsiveness in these mice exceeded the levels measured in animals sensitized without infection or infected without sensitization. The enhancement of AHR in mice sensitized and infected with RSV was associated with increased airway inflammation with bronchial exudates composed of mononuclear cells and eosinophils as well as massive tissue eosinophilia. Further, RSV infection resulted in increased levels of macrophage inflammatory protein $1 \alpha($ MIP-1 $1 \alpha)$ in the lungs of Df-sensitized mice and in a Th2 cytokine shift with increased production of IL-4 and IL-5 and a decrease in production of IFN- $\gamma$ by anti-CD3 
stimulated thoracic lymph node mononuclear cells. Increased levels of total serum IgE, but no changes in allergen-specific IgE or IgG1, were detectable. Measurements of RSV antigen by ELISA in lung homogenates revealed an increased virus load in sensitized compared to nonsensitized mice following tertiary infection.

These observations suggest that recurrent RSV infections augment the synthesis of Th2-derived cytokines, total $\mathrm{IgE}$ and MIP-1 $\alpha$ following allergen sensitization, thus inducing persistent and increased airway inflammation and AHR. In the absence of sensitization, the effects of RSV infection were attenuated on subsequent reinfection.

Hence, three studies indicate that there are consequences of respiratory virus infection in previously sensitized mice which lead to enhanced airway inflammation and increased AHR that can persist for long periods of time. Factors involved in this potentiation in allergic hosts could be increased production of chemokines in response to infection, increased recruitment of T-lymphocytes, possibly $\mathrm{Th} 2$, resulting in increased production of Th2-derived cytokines such as IL-4 and IL-5. These changes support an inflammatory response dominated by eosinophils as well as the development of persistent AHR (fig. 2). Less effective clearance of virus during an immune response with a Th2 bias may also have contributed to the changes seen. These findings in rodent models suggest that similar immune mechanisms may also be of importance in humans. On the one hand, allergic airway inflammation may, indeed, predispose to more severe obstructive airway disease following respiratory virus infection. On the other hand, respiratory virus infections are likely to play a role in both potentiation and perpetuation of airway

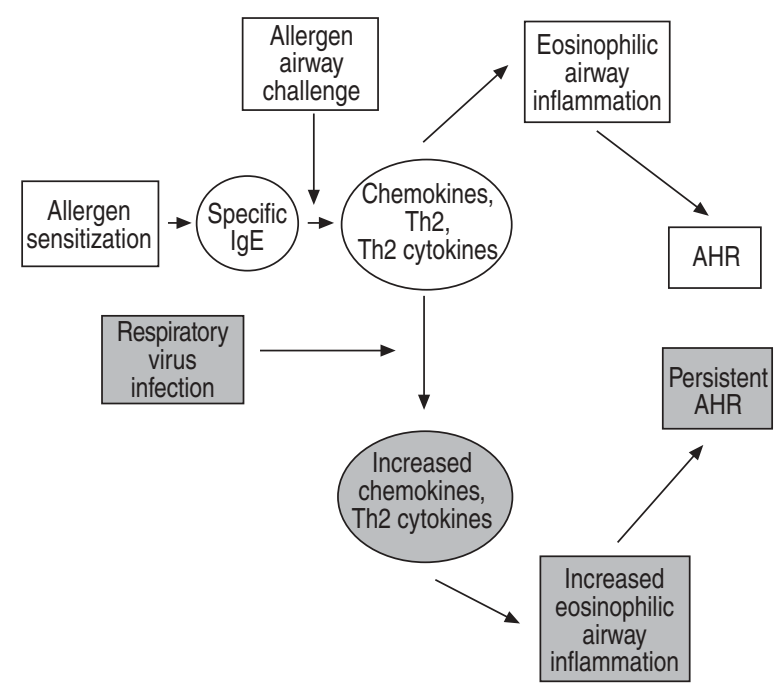

Fig. 2.-Potential mechanisms of enhanced airway inflammation and airway hyperresponsiveness following the allergic sensitization of the airways caused by respiratory virus infection in allergic mice ( $\square$ : allergic sensitization alone; :respiratory syncytial virus infection and its consequences if allergic airway inflammation preexists). IgE: immunoglobulin-E; Th2: type 2 T-helper cell; AHR: airway hyperresponsiveness. inflammation and airway obstruction in allergic asthma.

\section{Conclusion}

A number of animal models have provided evidence in favour of the following hypotheses, which are based on clinical and epidemiological observations. 1) Respiratory virus infections result in enhanced allergic sensitization to inhaled allergens and increase airway inflammation and obstruction following subsequent allergic airway sensitization. 2) Respiratory virus infections in the presence of allergic airway disease potentiate and perpetuate airway inflammation and obstructive symptoms. In addition, models were designed to define the pathogenetic mechanisms involved.

In the majority of the studies described earlier, respiratory virus infection enhanced sensitization to inhaled allergens and resulted in increased levels of antigen-specific antibodies. Allergic airway sensitization enhanced by prior virus infection can result in increased airway inflammation and heightened airway responsiveness, both features of bronchial asthma. In addition, respiratory viruses appear capable of enhancing the response to subsequent allergen sensitization independently of the levels of allergen-specific antibodies.

The mechanisms involved in this enhanced sensitization could be increased permeability of the airway mucosa to allergens and the recruitment of dendritic cells to the respiratory epithelium during acute respiratory virus infection. Further, suppression of tolerance induction to inhaled allergens during respiratory virus infections may contribute to enhanced allergic sensitization. Among the factors involved in augmenting the consequences of allergic airway sensitization are T-cells, especially CD8+ $\mathrm{T}$-cells as regulators of the responses, IL-5 as a pivotal cytokine required for the development of eosinophilic airway inflammation and eosinophils themselves, both effector cells of increased airway responsiveness and potential antigen-presenting cells. Depending on the timing of allergen exposure, respiratory virus infections which elicit a significant Th1-derived cytokine response may in turn inhibit allergic sensitization. These animal models support the hypothesis that respiratory virus infection can regulate allergic sensitization via the airways and the development of asthma.

In these models of respiratory virus infection in animals, the immune responses to allergen sensitization and the responses to infection interact and lead to increases in inflammation and responsiveness of the airways which persist for prolonged periods of time. These observations suggest that allergic airway sensitization and inflammation may indeed predispose to more severe disease following respiratory virus infection and that respiratory viruses may delay resolution and contribute to the perpetuation of allergic airway inflammation and obstructive airway symptoms.

The findings in rodent models may have their 
counterparts in human disease. The extent to which these models reflect human immunopathology remains to be assessed. Animal models have proved valuable in generating a number of reasonable pathogenetic concepts and can be used to address a host of unresolved questions regarding the immunology of respiratory virus infections, allergic sensitization and asthma. These include issues related to the possible persistence of virus, the role of the respiratory epithelium and of chemokines in the development of inflammation, the role of dendritic cells and eosinophils as antigen-presenting cells in the induction of T-cell responses, a definition of the link between inflammation (possibly eosinophils) and airway hyperresponsiveness, and the development of strategies to prevent the consequences of respiratory virus infections in allergic airway disease and asthma.

Acknowledgements. The authors would like to thank E. Hamelmann, K. Takeda, G Cieslewicz, M. Makela, A. Dakhama, M. Lahn and T. Ikemura for their collaboration, L.D. Shultz and M.C. Lamers for providing interleukin-4-, interferon gamma- and interleukin-5-deficient mice, respectively, and K.L. Bradley and A. Joetham for technical support.

\section{References}

1. Busse WW. Respiratory infections: their role in airway responsiveness and the pathogenesis of asthma. J Allergy Clin Immunol 1990; 85: 671-683.

2. Cypcar D, Stark J, Lemanske RF Jr. The impact of respiratory infections on asthma. Pediatr Clin North Am 1992; 39: 1259-1276.

3. Nicholson KG, Kent J, Ireland DC. Respiratory viruses and exacerbations of asthma in adults. $B M J$ 1993; 307: 982-986.

4. Johnston SL, Pattemore PK, Sanderson G, et al. Community study of role of viral infections in exacerbations of asthma in 9-11 year old children. BMJ 1995; 310: 1225-1229.

5. McIntosh K, Ellis EF, Hoffman LS, Lybass TG, Eller JJ, Fulginiti VA. The association of viral and bacterial respiratory infections with exacerbations of wheezing in young asthmatic children. J Pediatr 1973; 82: 578590.

6. Johnston SL. The role of viral and atypical bacterial pathogens in asthma pathogenesis. Pediatr Pulmonol Suppl 1999; 18: 141-143.

7. Frick OL, German DF, Mills J. Development of allergy in children. I. Association with virus infections. J Allergy Clin Immunol 1979; 63: 228-241.

8. Sims DG, Downham MA, Gardner PS, Webb JK, Weightman D. Study of 8-year-old children with a history of respiratory syncytial virus bronchiolitis in infancy. BMJ 1978; i: 11-14.

9. Webb MS, Henry RL, Milner AD, Stokes GM, Swarbrick AS. Continuing respiratory problems three and a half years after acute viral bronchiolitis. Arch Dis Child 1985; 60: 1064-1067.

10. Sly PD, Hibbert ME. Childhood asthma following hospitalization with acute viral bronchiolitis in infancy. Pediatr Pulmonol 1989; 7: 153-158.

11. Murray M, Webb MS, O'Callaghan C, Swarbrick AS, Milner AD. Respiratory status and allergy after bronchiolitis. Arch Dis Child 1992; 67: 482-487.

12. Sigurs N, Bjarnason R, Sigurbergsson F, Kjellman B, Bjorksten B. Asthma and immunoglobulin E antibodies after respiratory syncytial virus bronchiolitis: a prospective cohort study with matched controls. Pediatrics 1995; 95: 500-505.

13. Sigurs N, Bjarnason R, Sigurbergsson F, Kjellman B. Respiratory syncytial virus bronchiolitis in infancy is an important risk factor for asthma and allergy at age 7. Am J Respir Crit Care Med 2000; 161: 15011507.

14. Pullan CR, Hey EN. Wheezing, asthma, and pulmonary dysfunction 10 years after infection with respiratory syncytial virus in infancy. BMJ 1982; 284: $1665-1669$.

15. Kneyber MCJ, Steyerberg EW, de Groot R, Moll HA. Long-term effects of respiratory syncytial virus (RSV) bronchiolitis in infants and young children: a quantitative review. Acta Paediatr 2000; 89: 654-660.

16. Stein RT, Sherrill D, Morgan WJ, et al. Respiratory syncytial virus in early life and risk of wheeze and allergy by age 13 years. Lancet 1999; 354: 541-545.

17. Sakamoto M, Ida S, Takishima T. Effect of influenza virus infection on allergic sensitization to aerosolized ovalbumin in mice. J Immunol 1984; 132: 2614 2617.

18. Suzuki S, Suzuki Y, Yamamoto N, Matsumoto Y, Shirai A, Okubo T. Influenza A virus infection increases IgE production and airway responsiveness in aerosolized antigen-exposed mice. J Allergy Clin Immunol 1998; 102: 732-740.

19. Yamamoto N, Suzuki S, Shirai A, et al. Dendritic cells are associated with augmentation of antigen sensitization by influenza A virus infection in mice. Eur J Immunol 2000; 30: 316-326.

20. Holt PG, Vines J, Bilyk N. Effect of influenza virus infection on allergic sensitization to inhaled antigen in mice. Int Arch Allergy Appl Immunol 1988; 86: 121123.

21. Tsitoura DC, Kim S, Dabbagh K, Berry G, Lewis DB, Umetsu DT. Respiratory infection with influenza A virus interferes with the induction of tolerance to aeroallergens. J Immunol 2000; 165: 3484-3491.

22. Leibovitz E, Freihorst J, Piedra PA, Ogra PL. Modulation of systemic and mucosal immune responses to inhaled ragweed antigen in experimentally induced infection with respiratory syncytial virus. Implication in virally induced allergy. Int Arch Allergy Appl Immunol 1988; 86: 112-116.

23. Freihorst J, Piedra PA, Okamoto Y, Ogra PL. Effect of respiratory syncytial virus infection on the uptake of and immune response to other inhaled antigens. Proc Soc Exp Biol Med 1988; 188: 191-197.

24. O'Donnell DR, Openshaw PJ. Anaphylactic sensitization to aeroantigen during respiratory virus infection. Clin Exp Allergy 1998; 28: 1501-1508.

25. Miyajima I, Dombrowicz D, Martin TR, Ravetch JV, Kinet JP, Galli SJ. Systemic anaphylaxis in the mouse can be mediated largely through IgG1 and Fc $\gamma$ RIII. Assessment of the cardiopulmonary changes, mast cell degranulation, and death associated with active or IgE- or $\mathrm{IgG}_{1}$-dependent passive anaphylaxis. $\mathrm{J}$ Clin Invest 1997; 99: 901-914. 
26. Riedel F, Krause A, Slenczka W, Rieger CH. Parainfluenza-3-virus infection enhances allergic sensitization in the guinea-pig. Clin Exp Allergy 1996; 26: 603-609.

27. Dakhama A, Bramley AM, Chan NG, McKay KO, Schellenberg RR, Hegele RG. Effect of respiratory syncytial virus on subsequent allergic sensitization to ovalbumin in guinea-pigs. Eur Respir J 1999; 13: 976 982.

28. Lebrec H, Sarlo K, Burleson GR. Effect of influenza virus infection on ovalbumin-specific IgE responses to inhaled antigen in the rat. $J$ Toxicol Environ Health 1996; 49: 619-630.

29. Gershwin LJ, Himes SR, Dungworth DL, Giri SN, Friebertshauser KE, Camacho M. Effect of bovine respiratory syncytial virus infection on hypersensitivity to inhaled Micropolyspora faeni. Int Arch Allergy Immunol 1994; 104: 79-91.

30. Schwarze J, Hamelmann E, Bradley KL, Takeda K, Gelfand EW. Respiratory syncytial virus infection results in airway hyperresponsiveness and enhanced airway sensitization to allergen. J Clin Invest 1997; 100: 226-233.

31. Peebles RS Jr, Sheller JR, Johnson JE, Mitchell DB, Graham BS. Respiratory syncytial virus infection prolongs methacholine-induced airway hyperresponsiveness in ovalbumin-sensitized mice. $\mathrm{J}$ Med Virol 1999; 57: 186-192.

32. Matsuse H, Behera AK, Kumar M, Rabb H, Lockey RF, Mohapatra SS. Recurrent respiratory syncytial virus infections in allergen-sensitized mice lead to persistent airway inflammation and hyperresponsiveness. J Immunol 2000; 164: 6583-6592.

33. Kudlacz EM, Knippenberg RW. Parainfluenza virus type-3 infection attenuates the respiratory effects of antigen challenge in sensitized guinea pigs. Inflamm Res 1995; 44: 105-110.

34. Hamelmann E, Schwarze J, Takeda K, et al. Noninvasive measurement of airway responsiveness in allergic mice using barometric plethysmography. Am J Respir Crit Care Med 1997; 156: 766-775.

35. Foster PS, Hogan SP, Ramsay AJ, Matthaei KI,
Young IG. Interleukin 5 deficiency abolishes eosinophilia, airways hyperreactivity, and lung damage in a mouse asthma model. J Exp Med 1996; 183: 195201.

36. Hamelmann E, Oshiba A, Loader J, et al. Antiinterleukin-5 antibody prevents airway hyperresponsiveness in a murine model of airway sensitization. Am J Respir Crit Care Med 1997; 155: 819-825.

37. Hamelmann E, Cieslewicz G, Schwarze J, et al. Antiinterleukin 5 but not anti-IgE prevents airway inflammation and airway hyperresponsiveness. Am J Respir Crit Care Med 1999; 160: 934-941.

38. Schwarze J, Cieslewicz G, Hamelmann E, et al. IL-5 and eosinophils are essential for the development of airway hyperresponsiveness following acute respiratory syncytial virus infection. J Immunol 1999 162: 2997-3004.

39. Schwarze J, Cieslewicz G, Joetham A, et al. Critical roles for interleukin-4 and interleukin-5 during respiratory syncytial virus infection in the development of airway hyperresponsiveness after airway sensitization. Am J Respir Crit Care Med 2000; 162: 380-386.

40. Schwarze J, Makela M, Cieslewicz G, et al. Transfer of the enhancing effect of respiratory syncytial virus infection on subsequent allergic airway sensitization by T lymphocytes. J Immunol 1999; 163: 57295734.

41. Schwarze J, Cieslewicz G, Joetham A, Ikemura T, Hamelmann E, Gelfand EW. CD8 T cells are essential in the development of respiratory syncytial virusinduced lung eosinophilia and airway hyperresponsiveness. J Immunol 1999; 162: 4207-4211.

42. Hamelmann E, Oshiba A, Paluh J, et al. Requirement for CD8+ $\mathrm{T}$ cells in the development of airway hyperresponsiveness in a marine model of airway sensitization. J Exp Med 1996; 183: 17191729.

43. Robinson PJ, Hegele RG, Schellenberg RR. Allergic sensitization increases airway reactivity in guinea pigs with respiratory syncytial virus bronchiolitis. J Allergy Clin Immunol 1997; 100: 492-498. 